\title{
TIPE attenuates the apoptotic effect of radiation and cisplatin and promotes tumor growth via JNK and p38 activation in Raw264.7 and EL4 cells
}

\author{
YAO LIU* ${ }^{*}$ XIAO YAN NI* , RUI LING CHEN* , JUAN LI and FENG GUANG GAO \\ Department of Immunology, Basic Medical Sciences, Medical College, Xiamen University, Xiamen, Fujian 361102, P.R. China
}

Received November 16, 2017; Accepted March 30, 2018

DOI: $10.3892 /$ or.2018.6351

\begin{abstract}
Tumor necrosis factor $\alpha$-induced protein 8 (TIPE) is highly expressed in many types of malignancies. Apoptosis is the process of programmed cell death which maintains the balance of cell survival and death. TIPE is involved in the carcinogenesis of many tumor types, yet the exact role of TIPE in defective apoptosis-associated carcinogenesis remains uncertain. In the present study, TIPE-overexpressing Raw264.7 and EL4 cells and vector control cells were treated with $4 \mathrm{~mJ} / \mathrm{cm}^{2}$ ultraviolet radiation or $2 \mu \mathrm{g} / \mathrm{ml}$ cisplatin. Following ultraviolet irradiation, TIPE overexpression decreased the percentage of apoptotic cells as detected by flow cytometric and reversed the cisplatin-mediated decrease in mitochondrial membrane potential by JC-1 assay. Western blot analyses also revealed that TIPE overexpression inhibited cisplatin-induced activation of caspase- 3 and -9 and PARP. Secondly, TIPE overexpression increased the levels of phosphorylated JNK, MEK and p38. Moreover, inhibition of JNK and p38, but not MEK, efficiently abolished the cell pro-survival effect of TIPE. Most importantly, an in vivo tumor implantation model revealed that TIPE overexpression augmented the volume and weight of the implanted tumors, indicating that TIPE facilitated tumor formation. We found that TIPE exhibited an anti-apoptotic effect via JNK and p38 activation, which ultimately promoted tumor. Hence, the present study revealed that activation of JNK and p38 kinases contribute to the TIPE-mediated anti-apoptotic effect, indicating that JNK and p38 may be potential therapeutic molecules for TIPE overexpression-associated diseases.
\end{abstract}

Correspondence to: Professor Feng Guang Gao or Dr Juan Li, Department of Immunology, Basic Medical Sciences, Medical College, Xiamen University, Southern Xiang An Road, Xiamen, Fujian 361102, P.R. China

E-mail: gfengguang@xmu.edu.cn

E-mail: muzinvkouyue1983@163.com

*Contributed equally

Key words: TIPE, apoptosis, p38, JNK

\section{Introduction}

Tumor necrosis factor $\alpha$-induced protein 8 (TIPE, TNFAIP8) is a member of the TNFAIP8 family (1). TIPE is overexpressed in many types of malignancies, such as hepatocellular carcinoma, gastric adenocarcinoma, non-small cell lung cancer (NSCLC), breast and epithelial ovarian cancer (2-7). Apoptosis is the process of programmed cell death which maintains a healthy cell survival/cell death balance. Defects in the apoptotic process may cause cancer or autoimmunity, while enhanced apoptosis may cause degenerative diseases (8). In despite of the fact that TIPE is involved in the carcinogenesis of many tumor types (2-7), the exact role of TIPE in defective apoptosis-associated carcinogenesis is still uncertain.

The family of mitogen-activated protein kinases (MAPKs), which are specific to the amino acids serine and threonine, is involved in directing cellular responses to mitogens, osmotic stress and proinflammation (9). While the MAPK family regulates cell functions including proliferation, differentiation, mitosis and cell survival (1), it was documented that MAPK signaling, such as extracellular signal-regulated kinases (ERK), c-Jun N-terminal kinases (JNK) and p38 mitogen-activated protein kinases (p38) regulate apoptosis and play a prominent role in tumor development $(10,11)$. Blockade of p38 signaling was found to significantly inhibit the proliferation of head and neck squamous cell carcinoma cells (12), and p38 activation was also revealed to have counteracting effects with JNK on myeloid cell leukemia sequence (Mcl-1) expression and lead to pro-apoptotic effects in NSCLC cells (13). Nevertheless, JNK activation is essential for cell transformation and proliferation in response to Ras signaling (14). Hence, the role of MAPK activation in TIPE-associated carcinogenesis needs to be elucidated.

In the present study, we provide evidence that TIPE overexpression suppressed the apoptotic effect of radiation and cisplatin treatment in Raw264.7 and EL4 cells via JNK and p38 activation, which ultimately promoted tumor formation. This was supported by the fact that TIPE overexpression promoted the proliferation of Raw264.7 and EL4 cells. Secondly, TIPE overexpression increased the anti-apoptotic effects and the levels of phosphorylated JNK and p38. Moreover, inhibition of JNK and p38 efficiently abolished the pro-survival effects of TIPE. Most importantly, in vivo tumor implantation test revealed that TIPE overexpression obviously augmented the 
volume and weight of implanted tumors in mice, indicating that TIPE facilitated tumor formation. Hence, the present study revealed that activation of JNK and p38 kinases contributed to the TIPE-mediated anti-apoptotic effect, indicating that JNK and p38 may be potential therapeutic molecules for TIPE overexpression-associated diseases.

\section{Materials and methods}

Reagents and antibodies. Reagents were purchased from the following companies. Annexin V/PI apoptosis detection kit was obtained from Promega (Madison, WI, USA). JNK inhibitor SP600125 and p38 MAPK inhibitor SB203580 were obtained from Cayman Chemical (Ann Arbor, MI, USA) and used as previously described (15-17). Antibodies to $\beta$-actin (\#8457), proliferating cell nuclear antigen (PCNA) (\#13110), phospho(p)-p38 (\#4511), phosphorylated mitogen-activated protein kinase kinase (p-MEK) (\#9154), p-JNK (\#4668), cleaved caspase-3 (\#9664), cleaved caspase-9 (\#7237) and cleaved poly(ADP-ribose) polymerase (PARP) (\#5625) were purchased from Cell Signaling Technology, Inc. (Beverly, MA, USA). Antibody to TIPE (\#SC-82054) was purchased from Santa Cruz Biotechnology, Inc. (Dallas, TX, USA). The concentrations of the used antibodies were determined according to the manufacturer's instructions. The PrimeScript RT-PCR kit and SYBR Premix ExTaq ${ }^{\mathrm{TM}}$ kit were purchased from Tarkara Bio (Dalian, Liaoning, China). JC-1 (Molecular Probes, Eugene, OR, USA) was the dye used to detect early stage cell apoptosis. RPMI-1640 medium, Dulbecco's modified Eagle's medium (DMEM) and fetal bovine serum (FBS) were purchased from HyClone (GE Healthcare Life Sciences, Logan, UT, USA). UltraSensitive ${ }^{\mathrm{TM}}$ SP immunohistochemistry (IHC) kit was obtained from Maixin Biotech (Fuzhou, Fujian, China).

Animals. Pathogen-free BALB/c nude mice (5-6 weeks old, female) were obtained from the Shanghai Laboratory Animal Center of the Chinese Academy of Sciences and were housed at the Animal Center of Xiamen University under a $12 \mathrm{~h}$ light-dark cycle, temperature $\left(20-26^{\circ} \mathrm{C}\right)$ and humidity (40-70\%) conditions. They had free access to water and food. The animal study protocol was approved by the Committee on the Ethics of Animal Experiments of Xiamen University.

Cell lines. Abelson murine leukemia virus transformed Raw264.7 cells (18) and murine T lymphocyte EL4 cells which were transfected with Migrl control and TIPE vector were kindly provided by Professor Y.H. Chen (University of Pennsylvania, Philadelphia, PA, USA). Raw264.7 cells were cultured in DMEM with $10 \% \mathrm{FBS}$ at $37^{\circ} \mathrm{C}$ in $5 \% \mathrm{CO}_{2}$. EL4 cells were cultured in RPMI-1640 medium with $10 \%$ FBS at $37^{\circ} \mathrm{C}$ in $5 \% \mathrm{CO}_{2}$. Cells were synchronized by serum starvation for at least $6 \mathrm{~h}$ before further treatment.

Flow cytometric measurements. Cell apoptosis was determined as previously described (15). Briefly, TIPEoverexpressing and mock cells $\left(2 \times 10^{5} / \mathrm{ml}\right)$ were placed in 6 -well plates containing $10 \%$ FBS (HyClone) in phenol red-free RPMI-1640 (HyClone), were grown overnight, and washed once in PBS. Cells were irradiated with $4 \mathrm{~mJ} / \mathrm{cm}^{2}$ of
UVC light (HFsafe 1500; Class II, Type A2; Shanghai Lishen Scientific Equipment Co., Ltd., Shanghai, China), for $30 \mathrm{~min}$ as previously described (19). Then, the cells were collected and stained with Annexin V-FITC and propidium iodide for $20 \mathrm{~min}$ at room temperature. To determine the role of MAPK activation in TIPE-mediated increased cell pro-survival, the cells were pretreated with U0126, SP600125, SB203580 at a final concentration of $10 \mu \mathrm{M}$ prior to treatment with $2 \mu \mathrm{g} / \mathrm{ml}$ cisplatin, as previously described (15). Flow cytometry was performed using a FACSCalibur flow cytometer, and the data were analyzed using CellQuest software (BD Biosciences, San Jose, CA, USA).

Cell proliferation assay. Cell proliferation assay was performed as previously described (16). Briefly, 5x10 4 TIPEoverexpressing EL4 or Raw264.7 cells were inoculated in a 96-well plate at $100 \mu \mathrm{l} /$ well medium in a humidified incubator $\left(37^{\circ} \mathrm{C}, 5 \% \mathrm{CO}_{2}\right)$. Then, Cell Counting Kit-8 (CCK-8) solution $(10 \mu \mathrm{l})$ was added to each well of the plate and the OD450 value was determined at a wavelength of $450 \mathrm{~nm}$.

Measurement of mitochondrial membrane potential by flow cytometry. Mitochondrial membrane potential was assessed using the fluorescent dye JC-1, which detects the early stage of cell apoptosis (15). Briefly, TIPE-expressing EL4 and control cells were treated with $2 \mu \mathrm{g} / \mathrm{ml}$ cisplatin for $20 \mathrm{~min}$. Then, the cells were rinsed with PBS and JC-1 was added at a final concentration of $1 \mu \mathrm{g} / \mathrm{ml}$. After $15 \mathrm{~min}$ co-incubation at $37^{\circ} \mathrm{C}$, the cells were collected, washed twice with PBS and then analyzed by flow cytometry.

Reverse transcription PCR. The expression of TIPE in Raw264.7 and EL4 cells was investigated by RT-PCR analysis according to a previous description (20). Briefly, total RNA was isolated from the cells. Reverse transcription was performed using PrimeScript Reverse Transcriptase kit (Takara Biotechnology Co., Ltd., Dalian, China) and cDNA was used for subsequent PCR reactions using the Maxima SYBR-Green qPCR Master Mix (Takara Biotechnology Co., Ltd.). The following primers were used (Santa Cruz Biotechnology, Inc.): $\beta$-actin (sc-108070-PR), TNF $\alpha$-IP 8 (m)-PR (sc-76699-PR). However, primer sequences are not provided by Santa Cruz Biotechnology, Inc., as stated in their datasheets: 'Semi-quantitative RT-PCR may be performed to monitor TNF $\alpha$-IP 8 gene expression using RT-PCR Primer: TNF $\alpha$-IP 8 (m)-PR: sc-76699-PR, $\beta$-Actin (m)-PR: sc-108070-PR'.

Western blot analysis. The whole cellular protein was extracted and western blotting was performed as previously described (17,20). Briefly, proteins were obtained in lysis buffer and loaded onto sodiumdodecylsulfate-polyacrylamide gel electrophoresis (SDS-PAGE) gels for electrophoresis and transferred onto polyvinylidene difluoride (PVDF) membranes. After blocking in 5\% fat-free milk in Tris-buffered saline with Tween-20 (TBST) for $90 \mathrm{~min}$, the membranes were incubated with primary antibodies at $4^{\circ} \mathrm{C}$ overnight. Subsequently, the membranes were incubated with corresponding horseradish peroxidase (HRP)-conjugated secondary antibodies at room temperature for $90 \mathrm{~min}$. After washing 4 times with TBST 
A

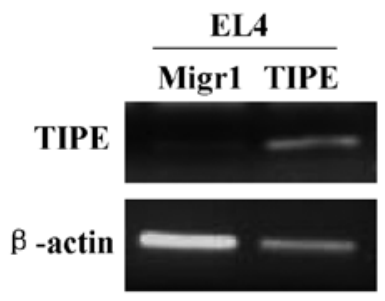

D

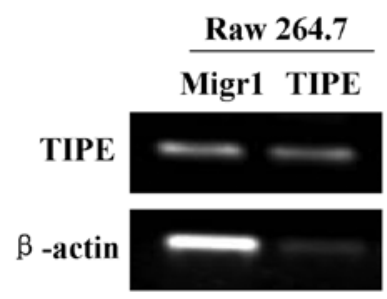

B
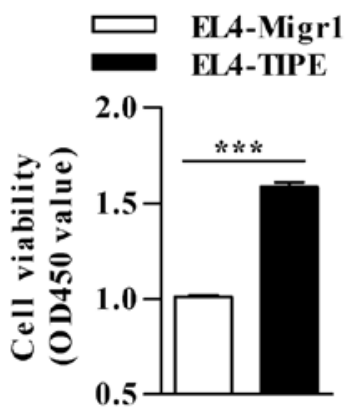

E

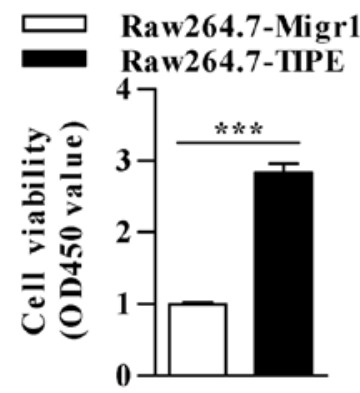

C

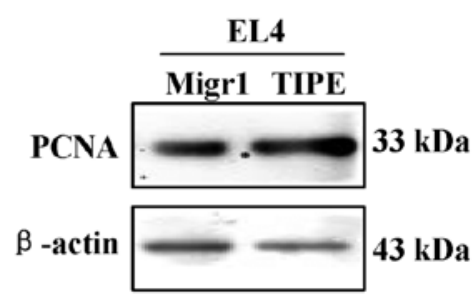

F

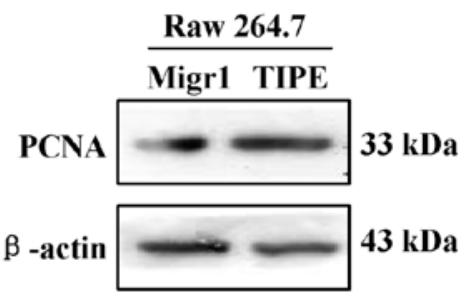

Figure 1. TIPE promotes proliferation of Raw264.7 and EL4 cells. TIPE was overexpressed in (A-C) EL4 and (D-F) Raw264.7 cells, and TIPE overexpression was confirmed by (A and D) RT-PCR. The effect of TIPE expression on cell proliferation was determined by (B and E) Cell Counting Kit-8 (CCK-8) assay and (C and F) western blotting of PCNA. For CCK-8 assays, data are presented as mean \pm SEM, $n=5 ;{ }^{* * *} \mathrm{P}<0.001$, Student's t-test. For RT-PCR and western blotting, $\beta$-actin was used as an internal control. A representative of 3 independent experiments is shown. TIPE, tumor necrosis factor $\alpha$-induced protein 8 .

(for 10 min each), the bound antibodies were visualized using enhanced chemiluminescence (ECL). $\beta$-actin was used as a loading control.

In vivo implantation tumor models. A xenograft tumor model in BALB/c mice was established as previously described (21) by subcutaneous neck injection of Raw264.7-TIPE and control cells $\left(2 \times 10^{6} /\right.$ nude mouse in $100 \mu 1$ volume). Tumor size was measured by vernier caliper (Shanghai Hui Yi Electronic Technology Co., Ltd., Shanghai, China), the length (L), width (W), and diameter were measured every two days from day 4 , tumor volume $\left(\mathrm{cm}^{3}\right)$ was calculated using the formula $\mathrm{W}^{2} \mathrm{x}(\mathrm{L} / 2)$. Following 12 days of growth, animals were sacrificed by $\mathrm{CO}_{2}$ asphyxiation and tumors were removed.

Immunohistochemical (IHC) staining. The expression of TIPE in the mouse tumor tissues was determined via IHC staining as previously described (20). Briefly, free-floating lung sections $(4 \mu \mathrm{M})$ were obtained using a slicing system (Leica RM2135). Endogenous peroxidase activity was quenched in $\mathrm{H}_{2} \mathrm{O}_{2}$, and $90 \%$ formic acid was used to expose the epitope. The primary antibody was applied overnight at $4^{\circ} \mathrm{C}$, and then biotinylated secondary antibody at room temperature. After a final wash, the slides were developed with diaminobenzidine substrate using the avidin-biotin HRP system.

Statistical analysis. All experiments were repeated at least 3 times to confirm the results. The data are presented as the mean \pm SEM. Student's t-test and one-way ANOVA with post Newman-Keuls test were applied. Differences were considered significant at $\mathrm{P}<0.05$.

\section{Results}

TIPE overexpression promotes the proliferation of Raw 264.7 and EL4 cells. The overexpression of TIPE in the Raw264.7 and EL 4 cells transfected with the TIPE-overexpression vector was confirmed by RT-PCR (Fig. 1A and D). Then, the effect of TIPE overexpression on cell proliferation was determined by CCK- 8 assays and western blotting, respectively. The results showed that TIPE overexpression increased the number of viable cells in the Raw264.7 and EL4 cell lines (Fig. 1B and E). The expression of PCNA was also upregulated in the TIPE-overexpressing cell lines (Fig. 1C and F). All these data indicate that TIPE has the ability to promote cell proliferation.

TIPE overexpression inhibits ultraviolet irradiation or cisplatin-induced apoptosis. To explore the effect of TIPE on cell apoptosis, TIPE-overexpressing Raw264.7 and EL4 cells were exposed to ultraviolet irradiation and the percentage of apoptotic cells was determined by flow cytometry. Under ultraviolet irradiated condition, TIPE overexpression exhibited a $\sim 18.5 \%$ anti-apoptotic ability in the EL4 cells, which was verified by the decreased percentage of apoptotic cells from 53.24 to $43.39 \%$ (Fig. 2A and B). Flow cytometric determination in Raw264.7 cells also revealed a similar trend (Fig. 2C and D). When JC-1 was used to measure mitochondrial membrane potential, TIPE overexpression obviously decreased the cisplatin-augmented mean fluorescence intensity (MFI) (Fig. 2E). Western blot analyses also revealed that TIPE overexpression inhibited cisplatin-induced activation of caspase-3, caspase-9 and PARP (Fig. 2F). All of these observations indicate that the cells acquired anti-apoptotic abilities following induction of TIPE overexpression. 
A

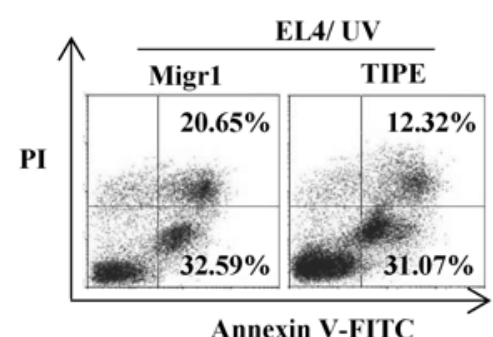

C
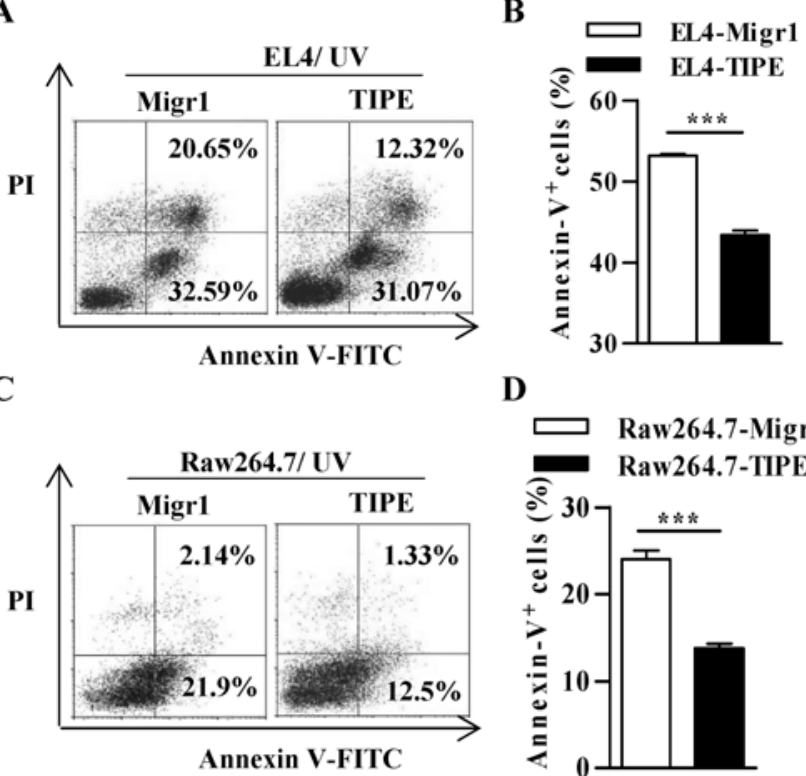

$\mathbf{E}$

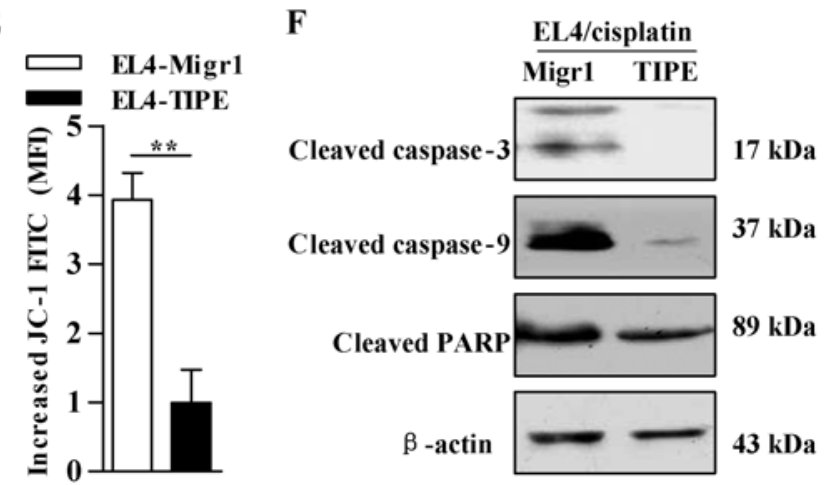

Figure 2. TIPE inhibits ultraviolet irradiation- or cisplatin-induced apoptosis in Raw264.7 and EL4 cells. (A-D) The cells were exposed to $4 \mathrm{~mJ} / \mathrm{cm}^{2}$ ultraviolet irradiation and cell apoptosis was determined by Annexin V/PI staining and flow cytometry. Data in the histograms represent the percentages of the analyzed population. Data are presented as mean \pm SEM, $n=3$, ${ }^{* * * *} \mathrm{P}<0.001$, Student's t-test. (E and F) Next, the cells were treated with $2 \mu \mathrm{g} / \mathrm{ml}$ cisplatin, and (E) mitochondrial membrane potential was determined by JC- 1 analyses, data are presented as mean \pm SEM, $n=3,{ }^{* *} \mathrm{P}<0.01$, Student's t-test and (F) levels of cleaved caspase-3, caspase-9 and PARP were determined by western blotting. For western blotting, $\beta$-actin was used as an internal control. A representative of 3 independent experiments is shown. TIPE, tumor necrosis factor $\alpha$-induced protein 8 . MFI, mean fluorescence intensity.

TIPE overexpression augments phosphorylation of JNK, p38 and $M E K$. MAPK was reported to regulate cell proliferation, differentiation and cell survival (1). The levels of phosphorylated MAPKs such as MEK, JNK, and p38 were determined to evaluate the role of MAPK in the TIPE-mediated increased cell pro-survival. An obvious increase in phosphorylated JNK, MEK and p38 was observed in the TIPE-overexpressing EL4 cells (Fig. 3A-C). A similar result was found in the TIPE-overexpressing Raw264.7 cells (Fig. 3D-F). All these data indicate that MAPK pathways may play an important role in the TIPE-mediated cell proliferation and anti-apoptotic effects.

Inhibition of 38 and JNK activation abolishes TIPE-induced anti-apoptotic effects. As TIPE overexpression efficiently
A

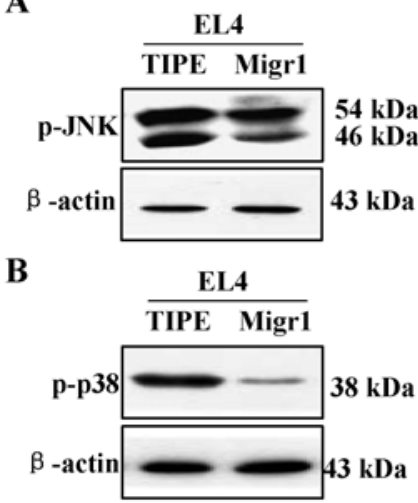

D

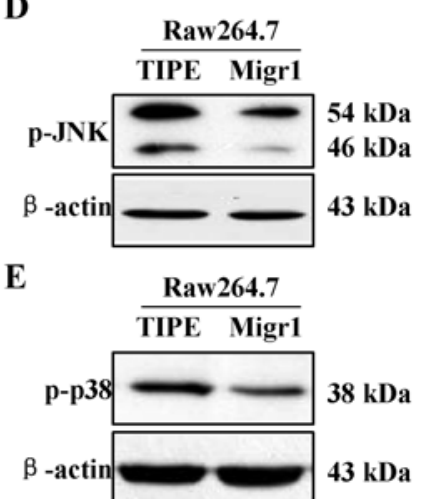

C

EL4

F

Raw264.7

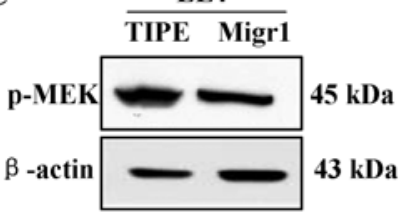

TIPE Migr1

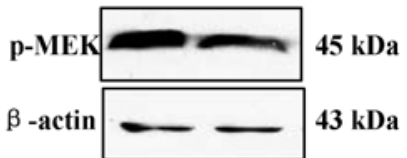

Figure 3. Overexpression of TIPE augments the phosphorylation of JNK, p38 and MEK. The levels of (A and D) phosphorylated (p)-JNK, (B and E) p-p38, and $(C$ and $D)$ p-MEK were determined by western blotting in the TIPE-overexpressing and Migr1 vector control EL4 and Raw264.7 cells. $\beta$-actin was used as an internal control. A representative of 3 independent experiments is shown. TIPE, tumor necrosis factor $\alpha$-induced protein 8 .

increased cell survival (Fig. 2) and obviously augmented MAPK activation (Fig. 3), we aimed to ascertain whether the TIPE-mediated anti-apoptotic effects were due to the activation of these kinases. Toward this end, TIPE-overexpressing and Migr1 control EL4 cells were incubated with related kinase inhibitors prior to cisplatin treatment and apoptotic cells were determined by flow cytometry. Compared with the Migrl control, TIPE overexpression efficiently inhibited cisplatin-induced cell apoptosis, which was confirmed by reduction in apoptotic cells from 58 to $41.18 \%$ (Fig. 4A and B). Compared with the U0126-treated group, inhibition of JNK phosphorylation by JNK inhibitor SP600125 efficiently abolished the promotive effect of TIPE on cell survival, which was confirmed by the increase in the percentages of apoptotic cells from 41.18 to $54.63 \%$ (Fig. 4). Treatment with SB203580 also showed a similar trend (Fig. 4). All these observations suggest that the activation of JNK, p38, but not MEK contributes to the anti-apoptotic effect of TIPE overexpression.

TIPE overexpression promotes tumor formation in vivo. We next constructed a tumor implantation model to observe the effect of TIPE overexpression on tumor formation. TIPE-overexpressing Raw264.7 and mock cells were subcutaneously implanted into BALB/c nude mice and the corresponding neoplasm volumes were measured every two days. TIPE overexpression not only increased tumor volume (Fig. 5A and B) but also augmented tumor weight (Fig. 5C). Immunohistochemical staining for TIPE in the implanted tumor tissues clearly showed that the transfected tumor cells consistently expressed TIPE (Fig. 5D). The results demonstrated that TIPE overexpression augmented tumor weight and volume indicating that TIPE overexpression facilitates tumor formation in vivo. 
A

EL4-Migr1/Cisplatin $\quad$ EL4-TIPE/Cisplatin

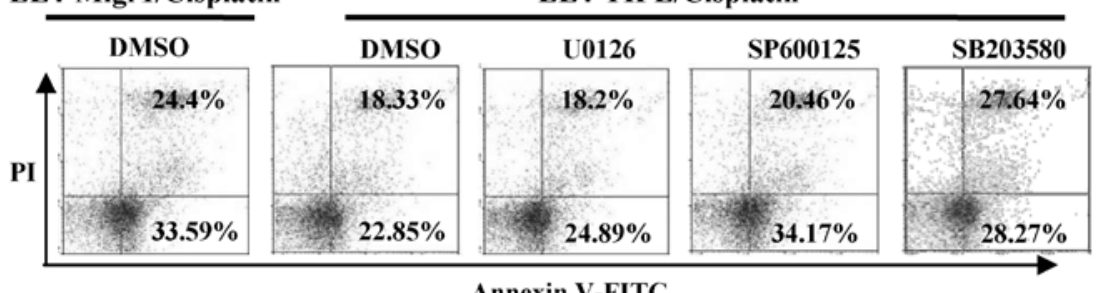

B

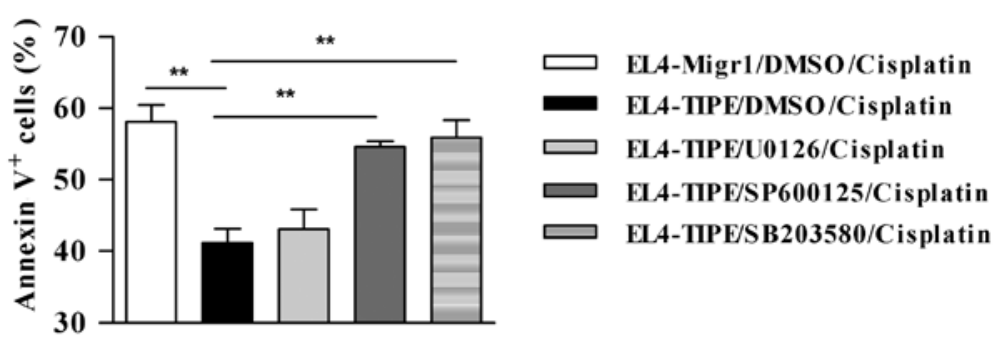

Figure 4. Inhibition of p38 and JNK activation abolishes TIPE-induced anti-apoptotic effects. (A and B) TIPE-overexpressing and Migrl control EL4 cells were treated with DMSO or U0126 (a highly selective inhibitor of both MEK1 and MEK2), SP600125 (JNK inhibitor) and SB203580 (p38 MAPK inhibitor) at the final concentration of $10 \mu \mathrm{M}$. Then, the cells were further incubated with $2 \mu \mathrm{g} / \mathrm{ml}$ cisplatin and cell apoptosis was assessed by flow cytometry with Annexin V/PI staining. Histogram data represent the positive percentages of the analyzed population. Data are presented as mean $\pm \mathrm{SEM}, \mathrm{n}=3,{ }^{* *} \mathrm{P}<0.01$, one-way ANOVA with post Newman-Keuls test. A representative of 3 independent experiments is shown. TIPE, tumor necrosis factor $\alpha$-induced protein 8 .

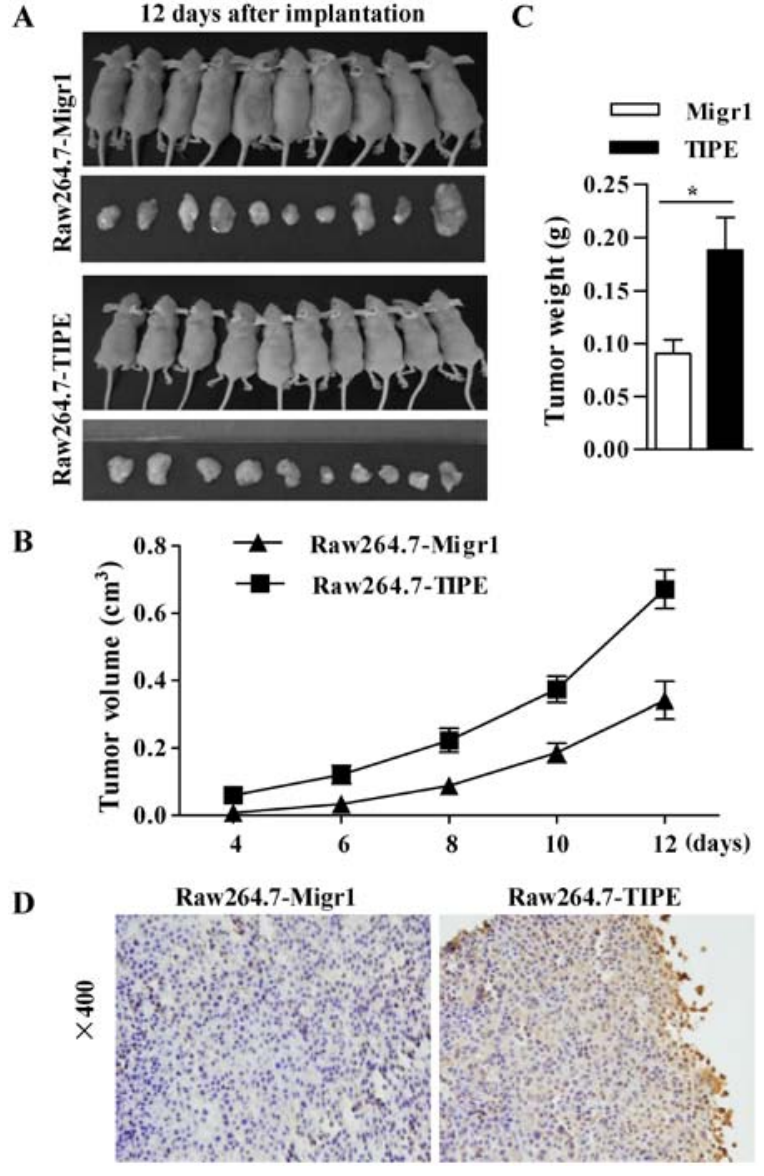

Figure 5. TIPE overexpression promotes tumor growth in vivo. TIPEoverexpressing Raw264.7 cells were subcutaneously implanted into $\mathrm{BALB} / \mathrm{c}$ nude mice. Tumor volume was measured and counted every 2 days. On day 12 , the mice were sacrificed and the tumor weight was evaluated. TIPE IHC staining was also performed. (A) Images of the mouse tumors. (B) The curve showing tumor growth (volume). (C) Mean weight of the tumors in both groups. (D) TIPE expression in the mouse tumor tissues. Data are presented as mean $\pm \mathrm{SEM}, \mathrm{n}=10 ;{ }^{*} \mathrm{P}<0.05$, Student's t-test. TIPE, tumor necrosis factor $\alpha$-induced protein 8 .

\section{Discussion}

In the present study, murine macrophage Raw264.7 and T lymphocyte EL4 cells were transfected with Migr1 control and a TIPE overexpression vector due to their abilities for suitable DNA transfection (18). In despite of the fact that TIPE is a newly described regulator of immunity and tumorigenesis and is the only known transfer protein of the lipid second messengers $(22,23)$, the exact mechanism of TIPE-mediated pro-survival effects remain uncertain. In the present study, we observed that TIPE inhibited cell apoptosis and promoted tumor formation in vivo. We found that TIPE overexpression augmented the levels of phosphorylated JNK and p38. Moreover, inhibition of JNK and p38 efficiently abolished the promotive effect of TIPE on cell survival. Most importantly, an in vivo tumor mouse model revealed that TIPE overexpression obviously augmented the volume and weight of the tumors, indicating that TIPE facilitates tumor formation.

Apoptosis can be initiated by two alternative signaling pathways: The death receptor-mediated extrinsic apoptotic and the mitochondrion-mediated intrinsic apoptotic pathway. Mitochondrial membrane potential reflects the pumping of hydrogen ions across the inner membrane (15). Cellular stress responses cause translocation of the Bcl-2 family from the cytosol to the mitochondria, resulting in decreased mitochondrial membrane potential, the release of cytochrome $c$, and the initiation of apoptosis (24). In the present study, we demonstrated that TIPE exhibited anti-apoptotic abilities, yet the exact effects of TIPE on mitochondrial membrane potential and the release of cytochrome $c$ need further investigation.

MAPK signaling, which includes ERK, p38 and JNK pathways, can be subverted to facilitate tumor proliferation, survival and invasion (25), indicating that MAPK signaling is involved in the process of carcinogenesis and tumor 
development. For example, an increased level of phosphorylated p38 was reported to be related to tumor size and the formation of satellite tumors (26). A higher activation of ERK was also found in many types of tumor (27). Activation of JNK signaling by the silencing of dual-specificity phosphatase 9 was documented to induce the proliferation of gastric cancer (28). Hence, in the present study, it was no surprise to find that the activation of MAPK signaling was upregulated by TIPE overexpression and promoted tumor formation. Notably, in spite of the fact that the activation of JNK and p38 contributed to the TIPE-induced anti-apoptotic effects, ERK phosphorylation had no effect on cell pro-survival. As prolonged JNK phosphorylation uncouples MEK-mediated ERK activation in a c-Jun-dependent manner (29), opposing effects of ERK and JNK-p38 MAPKs on apoptosis have been found in nerve growth factor withdrawal cells (30). Meanwhile, TIPE expression was found to be positively correlated with ERK1 but not ERK2 as documented in gastric cancer (31). Hence, the exact function of MEK phosphorylation in TIPE-mediated promotion of tumor formation remains unclear and needs further elucidation.

Mitochondrialintegrity plays animportant role in the process of cytochrome $c$ release and anti-apoptosis activity. Mcl-1, a member of the anti-apoptotic Bcl-2 family, was found to relocate into the mitochondrial membrane, antagonize Bcl-2-like protein 4 (Bax) and Bcl-2 homologous antagonist/killer (Bak) activation, and exert anti-apoptotics activity (32). The amelioration of mitochondrial membrane potential by regulating MAPK signaling was also demonstrated to exert the ability of pro-survival (33). Hence, the activation of JNK and p38 signaling by TIPE overexpression may have potential positive effects on the mitochondrial relocation of the components of the Bcl-2 family, maintain mitochondrial integrity, and exert pro-survival ability.

Taken together, our studies revealed that the activation of JNK and p38 kinases contributed to the TIPE-mediated anti-apoptotic effects and tumor formation, indicating that JNK and p38 may be potential therapeutic molecules for TIPE overexpression-associated diseases.

\section{Acknowledgements}

TIPE-overexpressing RAW264.7 and EL4 cells were supported by grant from National Institutes of Health, USA (AI-077533, AI-050059, and GM-085112 to YHC) and kindly provided by Professor YH Chen (University of Pennsylvania, Philadelphia, PA, USA).

\section{Funding}

This study was supported by grants from the National Natural Science Foundation of China (nos. 81273203 and 81771669), a grant from the Natural Science Foundation of Fujian Province of China (no. 2017J05140) and a grant from the Fujian Provincial Department of Education (JA15014).

\section{Availability of data and materials}

The datasets used during the present study are available from the corresponding author upon reasonable request.

\section{Author's contributions}

FGG designed the research and edited the manuscript. YL performed the western blotting and analyzed the data. XYN contributed to the flow cytometric analyses and CCK-8 assays and analyzed the data; RLC and JL contributed to manuscript preparation. All authors read and approved the manuscript and agree to be accountable for all aspects of the research in ensuring that the accuracy or integrity of any part of the work are appropriately investigated and resolved.

\section{Ethics approval and consent to participate}

The animal study protocol was approved by the Committee on the Ethics of Animal Experiments of Xiamen University.

\section{Consent for publication}

Not applicable.

\section{Competing interests}

All authors declare no competing interests.

\section{References}

1. Lou Y and Liu S: The TIPE (TNFAIP8) family in inflammation, immunity, and cancer. Mol Immunol 49: 4-7, 2011

2. Dong Q, Fu L, Zhao Y, Xie C, Li Q and Wang E: TNFAIP8 interacts with LATS1 and promotes aggressiveness through regulation of Hippo pathway in hepatocellular carcinoma. Oncotarget 8: 15689-15703, 2017.

3. Hadisaputri YE, Miyazaki T, Suzuki S, Yokobori T, Kobayashi T, Tanaka N, Inose T, Sohda M and Kuwano H: TNFAIP8 overexpression: Clinical relevance to esophageal squamous cell carcinoma. Ann Surg Oncol 19: S589-S596, 2012.

4. Hu R, Qiu X, Hong S, Meng L, Hong X, Qiu J, Yang J, Zhuang G and Liu Z: Clinical significance of TIPE expression in gastric carcinoma. Onco Targets Ther 9: 4473-4481, 2016.

5. Dong QZ, Zhao Y, Liu Y, Wang Y, Zhang PX, Jiang GY, Dong XJ, Cui QZ and Wang EH: Overexpression of SCC-S2 correlates with lymph node metastasis and poor prognosis in patients with non-small-cell lung cancer. Cancer Sci 101: 1562-1569, 2010.

6. Xiao M, Xu Q, Lou C, Qin Y, Ning X, Liu T, Zhao X, Jia S and Huang Y: Overexpression of TNFAIP8 is associated with tumor aggressiveness and poor prognosis in patients with invasive ductal breast carcinoma. Hum Pathol 62: 40-49, 2017.

7. Liu T, Gao H, Chen X, Lou G, Gu L, Yang M, Xia B and Yin H: TNFAIP8 as a predictor of metastasis and a novel prognostic biomarker in patients with epithelial ovarian cancer. Br J Cancer 109: 1685-1692, 2013.

8. Hassan M, Watari H, AbuAlmaaty A, Ohba Y and Sakuragi N: Apoptosis and molecular targeting therapy in cancer. Biomed Res Int 2014: 150845, 2014.

9. Pearson G, Robinson F, Beers Gibson T, Xu BE, Karandikar M, Berman K and Cobb MH: Mitogen-activated protein (MAP) kinase pathways: Regulation and physiological functions. Endocr Rev 22: 153-183, 2001

10. Huang P, Han J and Hui L: MAPK signaling in inflammation-associated cancer development. Protein Cell 1: 218-226, 2010.

11. Low HB and Zhang Y: Regulatory roles of MAPK phosphatases in cancer. Immune Netw 16: 85-98, 2016.

12. Leelahavanichkul K, Amornphimoltham P, Molinolo AA, Basile JR, Koontongkaew S and Gutkind JS: A role for p38 MAPK in head and neck cancer cell growth and tumor-induced angiogenesis and lymphangiogenesis. Mol Oncol 8: 105-118, 2014.

13. Azijli K, Yuvaraj S, van Roosmalen I, Flach K, Giovannetti E, Peters GJ, de Jong S and Kruyt FA: MAPK p38 and JNK have opposing activities on TRAIL-induced apoptosis activation in NSCLC H460 cells that involves RIP1 and caspase- 8 and is mediated by Mcl-1. Apoptosis 18: 851-860, 2013. 
14. Dérijard B, Hibi M, Wu IH, Barrett T, Su B, Deng T, Karin M and Davis RJ: JNK1: A protein kinase stimulated by UV light and Ha-Ras that binds and phosphorylates the c-Jun activation domain. Cell 76: 1025-1037, 1994.

15. Ke SZ, Ni XY, Zhang YH, Wang YN, Wu B and Gao FG: Camptothecin and cisplatin upregulate ABCG2 and MRP2 expression by activating the $\mathrm{ATM} / \mathrm{NF}-\kappa \mathrm{B}$ pathway in lung cancer cells. Int J Oncol 42: 1289-1296, 2013.

16. Wang YY, Liu Y, Ni XY, Bai ZH, Chen QY, Zhang Y and Gao FG: Nicotine promotes cell proliferation and induces resistance to cisplatin by $\alpha 7$ nicotinic acetylcholine receptor-mediated activation in Raw264.7 and El4 cells. Oncol Rep 31: 1480-1488, 2014.

17. Jin HJ, Li HT, Sui HX, Xue MQ, Wang YN, Wang JX and Gao FG: Nicotine stimulated bone marrow-derived dendritic cells could augment HBV specific CTL priming by activating PI3K-Akt pathway. Immunol Lett 146: 40-49, 2012.

18. Hartley JW, Evans LH, Green KY, Naghashfar Z, Macias AR, Zerfas PM and Ward JM: Expression of infectious murine leukemia viruses by RAW264.7 cells, a potential complication for studies with a widely used mouse macrophage cell line. Retrovirology 5: 1, 2008.

19. Kimura H, Lee C, Hayashi K, Yamauchi K, Yamamoto N, Tsuchiya H, Tomita K, Bouvet M and Hoffman RM: UV light killing efficacy of fluorescent protein-expressing cancer cells in vitro and in vivo. J Cell Biochem 110: 1439-1446, 2010.

20. Jiang YN, Yan HQ, Huang XB, Wang YN, Li Q and Gao FG: Interleukin 6 trigged ataxia-telangiectasia mutated activation facilitates lung cancer metastasis via MMP-3/MMP-13 up-regulation. Oncotarget 6: 40719-40733, 2015.

21. Williams ES, Rodriguez-Bravo V, Chippada-Venkata U, De Ia Iglesia-Vicente J, Gong Y, Galsky M, Oh W, Cordon-Cardo C and Domingo-Domenech J: Generation of prostate cancer patient derived xenograft models from circulating tumor cells. J Vis Exp 20: 53182, 2015.

22. Kumar D, Gokhale P, Broustas C, Chakravarty D, Ahmad I and Kasid U: Expression of SCC-S2, an antiapoptotic molecule, correlates with enhanced proliferation and tumorigenicity of MDA-MB 435 cells. Oncogene 23: 612-616, 2004.
23. Goldsmith JR and Chen YH: Regulation of inflammation and tumorigenesis by the TIPE family of phospholipid transfer proteins. Cell Mol Immunol 14: 482-487, 2017.

24. Czabotar PE, Lessene G, Strasser A and Adams JM: Control of apoptosis by the BCL-2 protein family: Implications for physiology and therapy. Nat Rev Mol Cell Biol 15: 49-63, 2014.

25. Wagner EF and Nebreda AR: Signal integration by JNK and p38 MAPK pathways in cancer development. Nat Rev Cancer 9: 537-549, 2009

26. Wang SN, Lee KT, Tsai CJ, Chen YJ and Yeh YT: Phosphorylated p38 and JNK MAPK proteins in hepatocellular carcinoma. Eur J Clin Invest 42: 1295-1301, 2012.

27. Samatar AA and Poulikakos PI: Targeting RAS-ERK signalling in cancer: Promises and challenges. Nat Rev Drug Discov 13: 928-942, 2014.

28. Wu F, Lv T, Chen G, Ye H, Wu W, Li G and Zhi FC: Epigenetic silencing of DUSP9 induces the proliferation of human gastric cancer by activating JNK signaling. Oncol Rep 34: 121-128, 2015.

29. Shen YH, Godlewski J, Zhu J, Sathyanarayana P, Leaner V, Birrer MJ, Rana A and Tzivion G: Cross-talk between JNK/SAPK and ERK/MAPK pathways: Sustained activation of JNK blocks ERK activation by mitogenic factors. J Biol Chem 278: 26715-26721, 2003

30. Xia Z, Dickens M, Raingeaud J, Davis RJ and Greenberg ME: Opposing effects of ERK and JNK-p38 MAP kinases on apoptosis. Science 270: 1326-1331, 1995.

31. Hu R, Liu W, Qiu X, Lin Z, Xie Y, Hong X, Paerhati R, Qi Z, Zhuang G and Liu Z: Expression of tumor necrosis factor- $\alpha$-induced protein 8 in stage III gastric cancer and the correlation with DcR3 and ERK1/2. Oncol Lett 11: 1835-1840, 2016.

32. Perciavalle RM, Stewart DP, Koss B, Lynch J, Milasta S, Bathina M, Temirov J, Cleland MM, Pelletier S, Schuetz JD, et al: Anti-apoptotic MCL-1 localizes to the mitochondrial matrix and couples mitochondrial fusion to respiration. Nat Cell Biol 14: $575-583,2012$.

33. Yu D, Li M, Tian Y, Liu J and Shang J: Luteolin inhibits ROS-activatedMAPK pathway in myocardial ischemia/reperfusion injury. Life Sci 122: 15-25, 2015. 